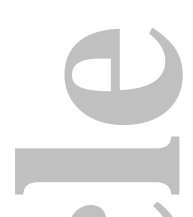

\title{
Two-year Clinical Outcomes of Patients Treated with Overlapping Absorb Scaffolds: An Analysis of the ABSORB EXTEND Single-Arm Study

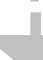

J Ribamar Costa Jr. MD ${ }^{1}$, Alexandre Abizaid MD, $\mathrm{PhD}^{1,2}$; Antonio L. Bartorelli MD ${ }^{3}$, Robert Whitbourn MD ${ }^{4}$ Patrick W. Serruys ${ }^{5}, \mathrm{MD}, \mathrm{PhD}$; Pieter C. Smits, $\mathrm{MD}^{6}$ on behalf of the ABSORB EXTEND investigators

${ }^{1}$ Instituto de Cardiologia Dante Pazzanese, Sao Paulo, Brazil ; ${ }^{2}$ Hospital Sirio Libanes, , Sao Paulo, Brazil, ${ }^{3}$ Centro Cardiologico Monzino, IRCCS, University of Milan, Milan, Italy, ${ }^{4} \mathrm{St}$ Vincent's Hospital, Fitzroy, Victoria, Australia, ${ }^{5}$ International Centre for Circulatory Health, NHLI, Imperial College London, London, UK ${ }^{6}$ Maasstad Hospital, Rotterdam, The Netherlands

Short title: Outcomes of ABSORB in overlapping

Conflict of interest: None of the authors have conflict to declare

Word count: 4463

\section{Correspondence to:}

Alexandre Abizaid., MD, PhD, FACC

Av. Dr. Dante Pazzanese, 500 - Vila Mariana - São Paulo, SP, Brasil

CEP 04012-180

e-mail: aabizaid@uol.com.br

This is the author manuscript accepted for publication and has undergone full peer review but has not been through the copyediting, typesetting, pagination and proofreading process, which may lead to differences between this version and the Version record. Please cite this article as doi:10.1002/ ccd.27223. 


\section{ABSTRACT}

Background: Pre-clinical data showed that overlapping (OVP) scaffolds might result in delayed healing and strut coverage compared to non-OVP scaffold segments. Furthermore, OVP in patients could result in increased peri-procedure myocardial infarction (MI) rate secondary to side branch occlusion; however, little is known whether this may have an impact on long-term clinical outcomes.

Methods: ABSORB EXTEND is a prospective, single-arm, open-label clinical study in which 812 patients were enrolled at 56 sites. In the present study, we compared the immediate and two-year clinical outcomes of patients with OVP scaffolds $(n=115)$ to those of patients with non-OVP scaffolds $(n=697)$. The primary objective was the comparison of MACE (cardiac death, MI and ischemic-driven TLR) and scaffold thrombosis (ST) rates up to 2 years.

Results: Baseline clinical and angiographic characteristics were comparable between cohorts except for longer lesions in the OVP patients as expected $(16.7 \pm 7.3$ vs. $11.6 \pm 4.4 \mathrm{~mm}$, p $<0.0001$ ), higher lesion complexity (B2) and numerically smaller vessel size. In-hospital, there was a marked increase in MACE in the OVP cohort (7.0\% vs. $0.9 \%, p=0.002)$, exclusively driven by a higher rate of peri-procedure MI $(7.0 \%$ vs. $0.9 \%, p=0.002)$. Longterm MACE did not significantly differ between groups (10.4\% in the OVP cohort vs. $6.6 \%$ in the no-OVP group, $\mathrm{p}=0.1)$ with comparable rates of cardiac death $(0.9 \%$ vs. $1.2 \%, \mathrm{p}=1.0)$ and ischemia-driven TLR (1.7\% vs. $2.5 \%, \mathrm{p}=1.0)$. Cumulative incidence of MI was higher in the OVP cohort $(7.8 \%$ vs. $3.0 \%, p=0.02)$. Of note, the rate of MI between hospital discharge and two-year follow-up was lower in the OVP cohort $(0.8 \%$ vs. $2.1 \%, p=0.04)$. Cumulative incidence of definite/probable ST was relatively low and comparable between groups $(1.8 \%$ vs. $1.5 \%, \mathrm{p}=0.7)$.

Conclusions: In this low-to-moderate complex population treated with the ABSORB scaffold the OVP group showed a higher incidence of peri-procedure MI with no immediate or long-term increase in cardiac death, TLR or ST.

Key words: ABSORB, overlapping, MACE, single arm study. 


\section{Introduction}

It is well established in the literature that metallic stent overlapping (OVP) is associated with higher peri-procedural myocardial infarction (MI), probably due to more side branch occlusion along the treated segment, an event linked also to the device design and strut thickness and width ${ }^{1,2}$.

The advent of metallic drug-eluting stents (DES) increased the concern related to the double layer of polymer and double dose of the anti-proliferative drug accrued in the OVP segments. Finn et al. analyzed rabbit models treated with $1^{\text {st }}$-generation DES (Cypher ${ }^{\mathrm{TM}}$ [Cordis Corporation, NJ, USA] and Taxus ${ }^{\mathrm{TM}}$ [Boston Scientific, MA, USA]) and their analogous bare-metal stents $\left(B x V e l o c i t y{ }^{\mathrm{TM}}\right.$ [Cordis Corporation] and Express ${ }^{\mathrm{TM}}$ [Boston Scientific]) and showed that, compared with bare-metal stents, $1^{\text {st }}$-generation DES further delay arterial healing and promote inflammation at sites of $\mathrm{OVP}^{3}$. Newer-generation DES, with thinner struts, open-cell design and more biocompatible or even biodegradable polymer coatings, contributed to minimize the negative impact of OVP and have been proven to be safer than $1^{\text {st }}$-generation $\mathrm{DES}^{4,5}$.

In theory, the recent introduction of BVS has made OVP more attractive, since these devices will be ultimately absorbed and the functionality of the treated segment should be potentially restored. Nonetheless, it is important to keep in mind that the current generation of BVS is still bulky with strut thickness and width of about150 $\mu \mathrm{m}$ and $200 \mu \mathrm{m}$, respectively, which are higher than those of the $1^{\text {st }}$-generation DES and may affect acute and long-term outcomes of this novel technology. In the present manuscript, we sought to compare acute and two-year outcomes of patients treated with ABSORB BVS with and without OVP scaffolds.

\section{Methods}

\section{Study Design and Population}

In the present analysis, we included all patients enrolled in the ABSORB EXTEND study. For comparison purposes, the entire population was divided according to the presence or not of OVP.

The details of the ABSORB EXTEND trial have been described elsewhere ${ }^{6}$. In brief, this was a prospective, single-arm, open-label clinical trial that enrolled 812 patients at 56 
international sites outside of the US. Target arteries should have a reference vessel diameter (RVD) $\geq 2.0 \mathrm{~mm}$ and $\leq 3.8 \mathrm{~mm}$, a maximum lesion length of $\leq 28 \mathrm{~mm}$, a diameter stenosis $\geq 50 \%$ and $<100 \%$. A maximum of two de novo native coronary artery lesions could be treated, each located in a different major epicardial vessel. Major exclusion criteria included a recent MI ( $<72$ hours before the index procedure) and target lesions located in the left main or within an arterial or saphenous vein graft. Also excluded were lesions with excessive tortuosity and/or heavy calcification.

Abbott Vascular (Santa Clara, CA) funded the study. The research ethics committee of each participating institution approved the protocol and all enrolled patients provided written informed consent before inclusion. The study is registered on clinicaltrials.gov (unique identifier NCT01023789).

\section{Study Device}

The study device (Abbott Vascular, Santa Clara, CA) is the same as that used in the ABSORB Cohort B trial, and has been described in detail previously ${ }^{7,8}$. In brief, the balloon expandable Absorb BVS is comprised of a poly L-lactide (PLLA) backbone, coated with a matrix composed of the antiproliferative drug everolimus (Certican ${ }^{\circledR}$ Novartis Pharmaceuticals Corporation, Basel, Switzerland) and the polymer poly (D-lactide) (PDLLA) in a 1:1 ratio to form an amorphous drug-eluting coating matrix containing $100 \mu \mathrm{g}$ everolimus $/ \mathrm{cm}^{2}$. Both PLLA and PDLLA are fully bioresorbable; PDLLA is expected to be completely absorbed by the body in 9 months and PLLA in approximately 24-36 months. During the resorption process, ester bonds in the PLLA and PDLLA chains are hydrolyzed, and small particles ( $\leq 2 \mu \mathrm{m}$ in diameter) are phagocytosed by macrophages. The ultimate degradation product of both PLLA and PDLLA is lactic acid, which is biologically ubiquitous and metabolized via the Krebs cycle?.

\section{Study Procedure}

Patients were enrolled through an interactive voice response system (Oracle America, Inc. Woburn, MA), following confirmation of angiographic inclusion criteria and delivery of the Absorb BVS device beyond the guiding catheter. Enrolled patients were to remain in the study until completion of the required follow-up period.

A maximum of two de novo native coronary artery lesions could be treated, each located in a different major epicardial vessel. The recommended range for target vessel diameter was assessed in terms of online quantitative coronary angiography (QCA) or 
intravascular ultrasound parameters of distal Dmax and proximal Dmax, which refer to the maximum lumen diameter evaluated at the distal and proximal ends of the target segment to be scaffolded, respectively. Planned OVP of scaffolds was permitted in lesions $>22 \mathrm{~mm}$ and $\leq 28 \mathrm{~mm}$ in length, with an OVP ranging between $1 \mathrm{~mm}$ and $4 \mathrm{~mm}$. Although not mandatory, the "scaffold-to-scaffold" implantation technique was recommended to minimize OVP, which was achieved by placing the balloon marker of the proximal scaffold just before the scaffold marker of the distal scaffold ${ }^{10}$.

All target lesions were to be treated using standard interventional techniques with mandatory predilation and scaffold implantation at a pressure not exceeding the balloon rated burst pressure. Postdilatation was left to the discretion of the investigator; however, if performed, was to be done with a non-compliant balloon sized to fit within the boundaries of the scaffold $(\leq 0.5 \mathrm{~mm}$ of the nominal scaffold diameter).

All patients enrolled in the study were to be pre-treated with a loading dose of $\geq 300$ $\mathrm{mg}$ of clopidogrel and $\geq 300 \mathrm{mg}$ of aspirin, followed by $75 \mathrm{mg}$ of clopidogrel daily for a minimum of 6 months and $\geq 75 \mathrm{mg}$ of aspirin daily indefinitely.

\section{Source document verification}

Source document verification (SDV) was routinely performed in $100 \%$ of all reported events and $100 \%$ of patients through 30 -day follow-up. Subsequently, SDV was performed in a random $20 \%$ of patients for the remaining follow-up visits.

\section{Follow-up}

Assessment of anginal status, data collection of adverse events, details of any subsequent coronary interventions, and use and changes in concomitant medications were collected at 30 days ( \pm 7 days), 180 days ( \pm 14 days) and at 1 and 2 years ( \pm 28 days).

\section{Study Endpoints and Definitions}

In addition to acute success, which is comprised of clinical device (analyzed on a per lesion basis) and clinical procedure success (analyzed on a per subject basis), endpoints include the comparison of adjudicated scaffold thrombosis (ST), cardiac death, MI (target and non-target vessel), and revascularization (target lesion revascularization [TLR]/target 
vessel revascularization [TVR]/all revascularizations) rates. The composite rates of ischemiadriven major adverse cardiac events (ID-MACE), ischemia-driven target vessel failure (IDTVF), ischemia-driven target lesion revascularization (ID-TLR) and ischemia-driven target vessel revascularization (ID-TVR) were also compared between patients with and without OVP.

An independent Clinical Event Committee (CEC) adjudicated all study endpoint events according to either protocol definitions and/or the Academic Research Consortium (ARC) definitions. All adverse events were reported to an independent Data and Safety Monitoring Board (DSMB), which reviewed the data to identify any safety issues related to the conduct of the study.

Clinical device success was defined as successful delivery and deployment of the clinical investigation scaffold at the target lesion and successful withdrawal of the scaffold delivery system with attainment of a final residual stenosis $<50 \%$ by QCA or by visual estimation if QCA was unavailable. Standard predilation and postdilatation balloon catheters (if applicable) could be used. Bailout patients were included as device success only if the above criteria for clinical device success were met.

Clinical procedure success was defined as successful delivery and deployment of the clinical investigation scaffold at the target lesion and successful withdrawal of the scaffold delivery system with attainment of a final residual stenosis of $<50 \%$ by QCA or by visual estimation if QCA was unavailable, and/or using any adjunctive device without the occurrence of ID-MACE during hospital stay with a maximum of first seven days post index procedure. In a dual lesion setting, both lesions must have met clinical procedure success.

Cardiac death was defined as any death due to proximate cardiac cause (e.g., MI, lowoutput heart failure, fatal arrhythmia). Unwitnessed death and death of unknown cause were classified as cardiac death. This included all procedure-related deaths comprising those related to concomitant treatment.

Classification and criteria for MI diagnosis were defined according to the per protocol definition. Q-wave MI was the development of a new, pathological Q wave. Non-Q-wave MI was elevation of CK levels to $\geq$ two times the upper limit of normal with elevated CK-MB in the absence of new pathological Q waves.

Revascularization events were defined as: a) ID-MACE, composed of cardiac death, MI (Q-wave and non-Q-wave) and ID-TLR by CABG or PCI; b) ID-TVF, composed of cardiac death, MI (Q-wave and non-Q-wave); c) ID-TVR by CABG or PCI. 
ID-TLR was defined as any repeat percutaneous intervention of the target lesion or bypass surgery of the target vessel with either positive functional ischemia study, ischemic symptoms and angiographic minimal lumen diameter stenosis $\geq 50 \%$ by core laboratory QCA or revascularization of a target lesion with diameter stenosis $\geq 70 \%$ by core laboratory QCA without either ischemic symptoms or a positive functional study.

ID-TVR was defined as any repeat percutaneous intervention or surgical bypass of any segment of the target vessel with either positive functional ischemia study or ischemic symptoms and an angiographic minimal lumen diameter stenosis $\geq 50 \%$ by core laboratory or revascularization of a target vessel with diameter stenosis $\geq 70 \%$ by core laboratory QCA without either ischemic symptoms or a positive functional study.

ST was categorized as acute ( $<1$ day), subacute (1-30 days) and late ( $>30$ days), and was defined according to the ARC guidelines ${ }^{11}$ as follows: definite, acute coronary syndrome and angiographic or pathologic confirmation of scaffold thrombosis; probable, unexplained death $\leq 30$ days or target vessel MI without angiographic information; and possible, unexplained death $>30$ days after scaffold placement.

\section{Statistical Analysis}

The sample size for ABSORB EXTEND rather than being defined based on an endpoint hypothesis was chosen to provide information on device performance and should be seen as hypothesis generating. All analyses were performed on the per treatment evaluable population.

For the descriptive statistics, categorical data are presented as counts and percentages and continuous variables are presented as mean \pm standard deviation (SD). Categorical variables were compared by the Chi-square test or Fisher's test when the Cochran's rule was not met. Continuous variables were compared by the Student $t$ test. Time-to-event MACE is presented as a Kaplan-Meier curve.

To determine the independent predictors of MACE, TLR, MI and ST among patients treated with the ABSORB BVS, a multivariable logistic regression model was built using a stepwise (forward/backward) procedure, with independent variables entered into the model at the 0.20 significance level and removed at the 0.10 level. Variables were eligible for inclusion in the multivariable logistic regression model-building process if the variable was present for $90 \%$ of the subjects in the analyses, they had a p-value $<0.2$ from the univariable 
analysis, and, if they highly correlated with another variable $(r>0.5$ and $p<0.05)$, had the higher level of significance. Presence of OVP was included in the model.

A two-tailed $p$ value $<0.05$ was considered statistically significant. However, since $p$ values presented here are for exploratory analysis only, they should therefore be interpreted cautiously.

\section{RESULTS}

Of the 812 patients (874 lesions) enrolled in the ABSORB EXTEND study, 115 (128 lesions, 14.2\%) were treated with OVP. Patients had a mean age of 61 years and were predominantly men in both cohorts. Baseline clinical demographics (Table 1) did not significantly differ between patients with and without OVP, except for the initial clinical presentation, with stable coronary disease more prevalent among patients treated with OVP, while patients without OVP had more frequently acute coronary syndrome as the initial presentation.

Table 2 displays the relevant angiographic clinical characteristics. As expected, lesions in the OVP cohort were longer with a higher prevalence of increased complexity (B2 according to ACC/AHA classification) $(66 \%$ vs. $38 \%, \mathrm{p}<0.01)$. Predilation was performed in almost all patients in both cohorts, while postdilation was more frequently performed among patients with OVP (77.4\% vs. $68.2 \%, \mathrm{p}=0.05)$.

Procedure success was comparable between the cohorts. However, clinical success was slightly higher among patients without OVP, mainly due to more peri-procedure MI detected among them $(94.3 \%$ vs. $90.4 \%, \mathrm{p}<0.001)$ (Table 3).

Notably, if we consider the current definition of good scaffold implantation (PSP $=$ patients with pre-dilatation, and QCA RVD $\geq 2.25 \mathrm{~mm}-\leq 3.5 \mathrm{~mm}$, and post-dilatation performed at $\geq 18$ atm, with post-dilatation balloon diameter $>$ nominal scaffold diameter but $\leq$ nominal scaffold diameter $+0.5 \mathrm{~mm}$ ), this was achieved in only $20 \%$ of the OVP cohort and $13.7 \%$ of the control group $(\mathrm{p}=0.02)$

Incidence of other MACE components, including cardiac death and vessel revascularization as well as ST, TLF and TVF were comparable between groups at two-year follow-up (Tables 4 and 5). Of note, there was no case of definitive ST in the OVP cohort in the first year after the procedure, while in the non-OVP group a $1.1 \%$ rate was observed in the same period. 
Figure 1 shows the temporal distribution of MACE between the two groups. As noticed, most of the adverse events in the OVP population occurred within the in-hospital period.

There was no independent predictor of MACE in the overall population. However, a trend toward more events was observed among patients with small reference vessel diameter (OR 0.90 IC95\% [0.01, 1.04], $\mathrm{p}=0.054$ ) and in those with prior MI history (OR 3.18 IC 95\% $[0.93,10.84], p=0.064)$. The only independent predictor of MI was a prior history of MI (OR 5.15 , IC95\% [1.42, 18.70], $\mathrm{p}=0.012)$. Independent predictor of a new revascularization procedure was treatment of multivessel disease (OR 6.16, IC 95\% [1.72, 22.11], p=0.005). There were no independent predictors of ST or death.

\section{DISCUSSION}

To the best of our knowledge, this is the largest long-term follow-up of patients undergoing treatment with OVP scaffolds. The results of this study should be interpreted with caution due to the differences in baseline lesion characteristics between the two groups. The main findings are the following: 1) there was a higher incidence of peri-procedure MI in the OVP patients who, however, had longer lesions, numerically smaller RVD, and greater B2 lesion complexity and; 2) two-year MACE including cardiac death, ID-TLR and ST were similar between these low-to-moderate complexity patient groups.

Treatment of long lesions with BVS frequently require scaffold OVP, since the maximum length of the commercially available devices is $28 \mathrm{~mm}$. Development of longer devices, although possible, would face one of the current limitations of this technology. Since the device should not be postdilated more than $0.5 \mathrm{~mm}$ than its nominal diameter to prevent the risk of acute disruption, the development of longer scaffolds would make difficult to accommodate vessel tapering, which often results in differences $>0.5 \mathrm{~mm}$ between the proximal and distal reference vessel segment in the scenario of long lesions.

Furthermore, it is not always technically easy or feasible to advance such bulky devices into each other to achieve OVP segments, especially when treating tortuous, and/or calcified vessels or when an unplanned OVP at the distal edge of a previously implanted BVS is needed. Use of tension/pressure to advance the device may result in dislodgement and damage to its integrity.

Once OVP is achieved, a segment with a 300-400- $\mu$ m layer of polymer will be created, which might impair local flow dynamic ${ }^{12}$ and increase the risk of side branch occlusion. Muramatsu et al. have already demonstrated that deployment of the Absorb BVS 
without OVP was associated with a higher incidence of post procedural side-branch occlusion when compared with a thin-strut new-generation DES (Xience V, Abbott Vascular $)^{13}$. Despite the lack of abundant clinical data, we speculate that these findings may be aggravated in segments of double layers of BVS. In a recent publication of the ABSORB II trial, Ishibashi et al. reported that BVS OVP was the single independent predictor of per protocol peri-procedure MI (OR: 5.07, 95\% CI: 1.78 to $14.41, \mathrm{p}=0.002)^{14}$. A previous analysis of the ABSORB EXTEND study database pointed also to OVP as a predictor of peri-procedure $\mathrm{MI}^{15}$. The present analysis confirms this finding that is probably related to the occlusion of more side branches along the longer segments treated with OVP.

Regarding long-term outcomes, the presence of scaffold OVP has also been associated with negative findings, at least in the pre-clinical scenario. Farooq et al. examined 41 OVP performed with the ABSORB BVS or the everolimus-eluting metallic DES in pigs and showed that strut coverage at 28 days was delayed in the OVP scaffolds because of the overlay configuration of the thicker ABSORB BVS struts. However, at 90 days OVP of both ABSORB BVS and metallic DES showed comparable strut coverage ${ }^{16}$. It is noteworthy that the pre-clinical observation of delayed strut coverage did not translate into an increased occurrence of serious untoward events in our clinical evaluation. This might be related to adherence to the dual antiplatelet therapy (roughly $96 \%$ at 6 months and $79 \%$ at one year, for both groups), the frequent optimization of the procedure with postdilation (especially in the OVP group) and the low-to-moderate complexity of the enrolled population. Furthermore, in our present study, use of OVP also did not turn out as an independent predictor of MACE, TLF, MI, TLR or ST.

It is also important to bear in mind that this study was conceived and primarily conducted prior to the current recommendations for optimal scaffold deployment, including the endorsement for performing routine postdilation with high-pressure non-compliant balloons within the limits of expansion of the device, as recently published by Ortega-Paz et al. ${ }^{17}$.

Recently, Sotomi et al. published the results of 14 patients treated with ABSORB OVP and evaluated with OCT at two years ${ }^{18}$. They compared the results in the segments with and without OVP. Notably, lumen area and endoluminal scaffold areas were similar in both segments despite the neointimal area being larger in the overlap segments. The neointimal coverage was essentially fully complete in both non-overlap (99.4 $\pm 0.8 \%$ ) and overlap segments $(99.8 \pm 0.4 \%)$ at two-year follow-up. The flow area in the overlap segments was not different from the flow area in the non-overlap segments, despite the neointimal response 
being greater in the overlap segments. Consequently, the treated segments showed a homogeneous lumen area through the scaffold segment. These results might support the feasibility of overlapping scaffolds when needed for longer lesions if acute lumen expansion is achieved in a similar extent as in the non-overlap segments using good implantation techniques.

\section{LIMITATIONS}

This post-hoc analysis was performed from a non-randomized study. No correction has been made regarding differences in baseline characteristics between the two comparison groups. These differences could have heavily contributed to the results. The study was underpowered for low-frequency events, like ST. The implantation technique used in the ABSORB Extend was outdated. Indeed, the PSP (vessel predilation, sizing and postdilation)

implant strategy is highly recommended and followed today. Finally, the lack of a control arm with a metallic DES does not allow for comparison of the devices.

\section{CONCLUSIONS}

In this low-to-moderate complex population treated with the BVS in the ABSORB EXTEND study, scaffold OVP was associated with an increase of peri-procedure MI but without any immediate or long-term effect on cardiac death, TLR or ST occurrence.

\section{REFERENCES}

1. Ellis SG, Savage M, Fischman D et al. Restenosis after placement of PalmazSchatz stents in native coronary arteries. Initial results of a multicenter experience. Circulation 86(6), 1836-1844 (1992).

2. Kastrati A, Schömig A, Elezi S et al. Predictive factors ofrestenosis after coronary stent placement. J. Am. Coll. Cardiol.30(6), 1428-1436 (1997).

3. Finn AV, Kolodgie FD, Harnek $J$ et al. Differential response of delayed healing and persistent inflammation at sites of overlapping sirolimus- or paclitaxel-eluting stents. Circulation 112(2), 270-278 (2005).

4. Farooq V, Vranckx P, Mauri L et al. Impact of overlapping newer generation drug-eluting stents on clinical and angiographic outcomes: pooled analysis of five trials from the international Global RESOLUTE Program. Heart 99(9), 626-633 (2013). 
5. Kitabata H, Loh JP, Pendyala LK et al. Safety and efficacy outcomes of overlapping second-generation everolimus-eluting stents versus first-generation drug-eluting stents. Am. J.Cardiol. 112(8), 1093-1098 (2013).

Abizaid A, Ribamar Costa J Jr, Bartorelli AL, Whitbourn R, van Geuns RJ, Chevalier B, Patel T, Seth A, Stuteville M, Dorange C, Cheong WF, Sudhir K, Serruys PW; ABSORB EXTEND investigators. The ABSORB EXTEND study: preliminary report of the twelve-month clinical outcomes in the first 512 patients enrolled. EuroIntervention. 2015 Apr;10(12):1396-401.

7. Oberhauser JP, Hossainy S, Rapoza R. Design principles and performance of bioresorbable polymeric vascular scaffolds. EuroIntervention. 2009;Vol.5:F15-F22

8. Okamura T, Garg S, Gutierrez-Chico JL, Shin ES, Onuma Y, Garcia-Garcia HM, Rapoza RJ, Sudhir K, Regar E, Serruys PW. In vivo evaluation of stent strut distribution patterns in the bioabsorbable everolimus-eluting device: An oct ad hoc analysis of the revision 1.0 and revision 1.1 stent design in the absorb clinical trial. EuroIntervention. 2010;5:932-938

9. Serruys PW, Onuma Y, Ormiston JA, de Bruyne B, Regar E, Dudek D, Thuesen L, Smits PC, Chevalier B, McClean D, Koolen J, Windecker S, Whitbourn R, Meredith I, Dorange C, Veldhof S, Miquel-Hebert K, Rapoza R, García-García HM. Evaluation of the second generation of a bioresorbable everolimus drug-eluting vascular scaffold for treatment of de novo coronary artery stenosis: six-month clinical and imaging outcomes. Circulation. 2010 Nov 30;122(22):2301-12.

10. Rigatelli G, Avvocata FD, Ronco F, Giordan M, Roncon L, Caprioglio F, Grassi G, Faggian G, Cardaioli P. Edge-to-Edge Technique to Minimize Ovelapping of Multiple Bioresorbable Scaffolds Plus Drug Eluting Stents in Revascularization of Long Diffuse Left Anterior Descending Coronary Artery Disease. J Interv Cardiol. 2016 Jun;29(3):275-84.

11. Cutlip D, Windecker S, Mehran R, Boam A, Cohen D, van Es G-A, Steg P, Morel M-A, Mauri L, Vranckx P, McFadden E, Lansky A, Hamon M, Krucoff M, Serruys P, Consortium aobotAR. Clinical end points in coronary stent trials. A case for standardized definitions. Circulation. 2007;115:2344-2351

12. Rikhtegar F, Wyss C, Stok KS, Poulikakos D, Müller R, Kurtcuoglu V. Hemodynamics in coronary arteries with overlapping stents. J. Biomech. 47(2), 505-511 (2014). 
13. Muramatsu T, Onuma Y, García-García HM et al.; ABSORB EXTEND Investigators. Incidence and short-term clinical outcomes of small side branch occlusion after implantation of an everolimus-eluting bioresorbable vascular scaffold: an interim report of 435 patients in the ABSORB-EXTEND single-arm trial in comparison with an everolimuseluting metallic stent in the SPIRIT first and II trials. JACC Cardiovasc. Interv. 6(3), 247257 (2013).

14. Ishibashi Y, Muramatsu T, Nakatani S, Sotomi Y, Suwannasom P, Grundeken MJ, Cho YK, Garcia-Garcia HM, van Boven AJ, Piek JJ, Sabaté M, Helqvist S, Baumbach A, McClean D, de Sousa Almeida M, Wasungu L, Miquel-Hebert K, Dudek D, Chevalier B, Onuma Y, Serruys PW. Incidence and Potential Mechanism(s) of Post-Procedural Rise of Cardiac Biomarker in Patients With Coronary Artery Narrowing After Implantation of an Everolimus-Eluting Bioresorbable Vascular Scaffold or Everolimus-Eluting Metallic Stent. JACC Cardiovasc Interv. 2015 Jul;8(8):1053-63.

15. De Ribamar Costa Jr J, Abizaid A, Bartorelli A, Whitbourn R, Jepson N, Perin M, Steinwender C, Stuteville M, Ediebah D, Sudhir K, Serruys PW. One-year clinical outcomes of patients treated with everolimus-eluting bioresorbable vascular scaffolds versus everolimus-eluting metallic stents: a propensity score comparison of patients enrolled in the ABSORB EXTEND and SPIRIT trials. EuroIntervention 2016;12:1255-1262

15. Farooq V, Serruys PW, Heo JH et al. Intracoronary optical coherence tomography and histology of overlapping everolimus-eluting bioresorbable vascular scaffolds in a porcine coronary artery model: the potential implications for clinical practice. JACC Cardiovasc. Interv. 6(5), 523-532 (2013).

17. Ortega-Paz L, Capodanno D, Gori T, Nef H, Latib A, Caramanno G, Di Mario C, Naber C, Lesiak M, Capranzano P, Wiebe J, Mehilli J, Araszkiewicz A, Pyxaras S, Mattesini A, Geraci S, Naganuma T, Colombo A, Münzel T, Sabaté M, Tamburino C, Brugaletta S. Predilation, sizing and post-dilation scoring in patients undergoing everolimuseluting bioresorbable scaffold implantation for prediction of cardiac adverse events: development and internal validation of the PSP score. EuroIntervention. 2017 Apr 20;12(17):2110-2117.

18. Sotomi Y, Suwannasom P, Wu CJ, Tateishi H, Cheong WF, Zhao WF, Veldhof S, Winter RJ, Wykrzykowska J, Farooq V, Abizaid A, Serruys PW, Onuma Y. Imaging outcomes of bioresorbable scaffold overlap: an optical coherence tomography analysis from the ABSORB EXTEND trial. AsiaIntervention 2017;3:49-57. 


\section{Figure Legend}

Figure 1. Cumulative incidence of MACE in patients treated with and without ABSORB overlapping. On the left the two-year curve for both groups and, on the right, the landmark analysis with the in hospital events.
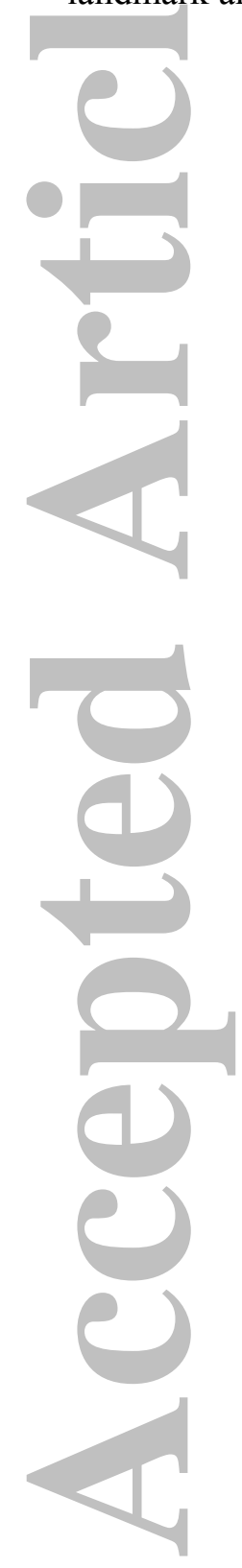
Table 1. Baseline characteristics of the study patients

\begin{tabular}{|c|c|c|c|c|}
\hline Variables & Total & OVP scaffolds & Non-OVP scaffolds & P-value* \\
\hline 1 & $\mathrm{~N}=812$ pts & $\mathrm{N}=115$ pts & $N=697$ pts & \\
\hline Age (years) & $61 \pm 4.3$ & $61 \pm 4.7$ & $61 \pm 3.9$ & 0.71 \\
\hline Gender male, n (\%) & $603(74)$ & $82(71)$ & $523(75)$ & 0.43 \\
\hline Active smoking, n (\%) & $468(58)$ & $66(57)$ & $402(58)$ & 0.95 \\
\hline Diabetes Mellitus, n (\%) & & $28(24)$ & $187(27)$ & 0.58 \\
\hline Hypertension**, n (\%) & $563(69)$ & $80(70)$ & $483(69)$ & 0.95 \\
\hline Hypercholesterolemia**, n (\%) & $550(68)$ & $82(71)$ & $468(67)$ & 0.38 \\
\hline CAD in family, $\mathrm{n}(\%)$ & $276(37)$ & $45(42)$ & $231(36)$ & 0.25 \\
\hline Prior MI, n (\%) & $230(29)$ & $31(27)$ & 199 (29) & 0.74 \\
\hline \multicolumn{5}{|l|}{ Clinical Presentation, n (\%) } \\
\hline Silent ischemia & $49(6)$ & $9(8)$ & $40(6)$ & 0.39 \\
\hline Stable angina & $461(57)$ & $83(72)$ & $378(54)$ & 0.00 \\
\hline - Unstable angina & $215(27)$ & $20(17)$ & $195(28)$ & 0.02 \\
\hline Non STEMI & $87(10)$ & $3(3)$ & $84(12)$ & $<0.01$ \\
\hline
\end{tabular}

OVP: overlapping

* Between OVP and non OVP

** Requiring medication 
Catheterization and Cardiovascular Interventions This article is protected by copyright. All rights reserved. 
Table 2. Angiographic and procedure characteristics

\begin{tabular}{|c|c|c|c|c|}
\hline 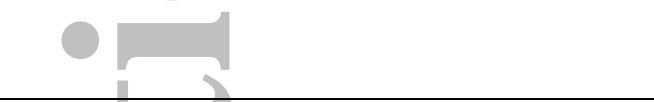 & $\begin{array}{l}\text { Total population } \\
\mathrm{N}=\mathbf{8 7 4} \text { lesions }\end{array}$ & $\begin{array}{l}\text { OVP scaffolds } \\
\mathrm{N}=128 \text { lesions }\end{array}$ & $\begin{array}{l}\text { Non-OVP scaffolds } \\
\mathrm{N}=746 \text { lesions }\end{array}$ & $P$-value \\
\hline \multicolumn{5}{|l|}{ Vessel treated, $\mathrm{n}(\%)$} \\
\hline - RCA (\%) & $250(29)$ & $39(31)$ & $211(28)$ & 0.61 \\
\hline$-\quad$ LAI & $395(45)$ & $67(52)$ & $328(44)$ & 0.08 \\
\hline$-\quad \operatorname{LCX}(\%)$ & $228(26)$ & $22(17)$ & $206(28)$ & 0.01 \\
\hline Tortuosity $>45^{\circ}, \mathrm{n}(\%)$ & $26(3)$ & $6(5)$ & $20(3)$ & 0.25 \\
\hline Calcification $\geq$ moderate, $\mathrm{n}(\%)$ & $121(14)$ & $23(18)$ & $98(13)$ & 0.25 \\
\hline Thrombus, $n$ (\%) & $14(2)$ & $2(2)$ & $12(2)$ & 1.00 \\
\hline Reference vessel diameter, mean \pm SD & $2.64 \pm 0.39$ & $2.59 \pm 0.34$ & $2.66 \pm 0.39$ & 0.06 \\
\hline Lesion length $(\mathrm{mm})$, mean $\pm \mathrm{SD}$ & $12.33 \pm 5.26$ & $16.7 \pm 7.32$ & $11.6 \pm 4.42$ & $<0.001$ \\
\hline \multicolumn{5}{|l|}{ ACC/AHA lesion classification, $n(\%)$} \\
\hline - Type A (\%) & $19(2)$ & $0(0)$ & $19(3)$ & 0.09 \\
\hline - Type B1 (\%) & $459(53)$ & $39(31)$ & $420(57)$ & $<0.001$ \\
\hline - Type B2 (\%) & $363(42)$ & $83(66)$ & $280(38)$ & $<0.001$ \\
\hline
\end{tabular}




$\begin{array}{lccc} & & & \\ \end{array}$


Table 3. In-hospital clinical outcomes

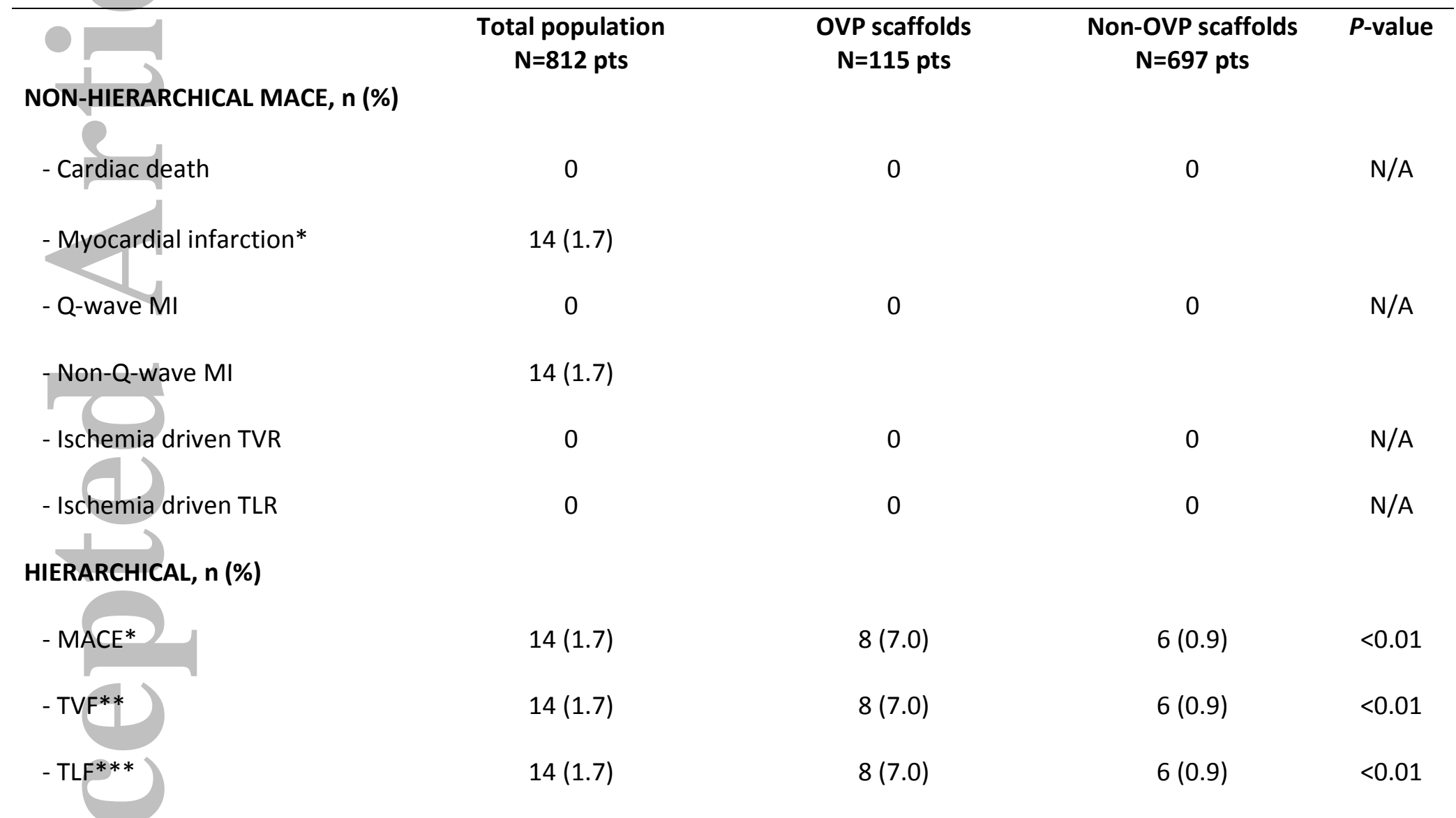

OVP: overlapping; MACE: major adverse cardiac events; MI: myocardial infarction; TVR: target vessel revascularization; TLR: target lesion revascularization; TVF: target vessel failure; TLF: target lesion failure.

*per protocol (please refer to study definition of $\mathrm{MI}$ in the methods section) 


\section{Table 4. Two-year clinical outcomes}

\begin{tabular}{|c|c|c|c|c|}
\hline & $\begin{array}{l}\text { Total population } \\
\quad \mathrm{N}=\mathbf{8 1 2} \text { pts }\end{array}$ & $\begin{array}{l}\text { OVP scaffolds } \\
\mathrm{N}=115 \text { pts }\end{array}$ & $\begin{array}{c}\text { Non-OVP scaffolds } \\
\mathrm{N}=697 \text { pts }\end{array}$ & $P$-value \\
\hline \multicolumn{5}{|c|}{ NON-HIERARCHICAL MACE, n (\%) } \\
\hline - Cardiac death & $9(1.1)$ & $1(0.9)$ & $8(1.2)$ & 1.0 \\
\hline - Myocardial infarction* & $35(12.3)$ & $10(8.7)$ & $25(3.6)$ & 0.02 \\
\hline - Q wave MI & $10(1.2)$ & $2(1.7)$ & $8(1.1)$ & 0.6 \\
\hline - Non Q wave MI & $25(3.1)$ & $8(6.9)$ & $17(2.4)$ & 0.02 \\
\hline - Ischemia driven TVR & $46(5.7)$ & $1(0.9)$ & $22(3.1)$ & 0.2 \\
\hline - Ischemia driven TLR & $34(4.2)$ & $1(0.9)$ & $18(2.6)$ & 0.5 \\
\hline \multicolumn{5}{|l|}{ HIERARCHICAL, n (\%) } \\
\hline - MACE* & $58(7.2)$ & $12(10.4)$ & $46(6.6)$ & 0.1 \\
\hline$-\mathrm{TVF}^{* *}$ & $68(8.4)$ & $13(11.3)$ & $55(7.9)$ & 0.2 \\
\hline$-\mathrm{TLF}^{* * *}$ & $57(7.1)$ & $12(10.4)$ & $45(6.5)$ & 0.1 \\
\hline
\end{tabular}

OVP: overlapping; MACE: major adverse cardiac events; MI: myocardial infarction; TVR: target vessel revascularization; TLR: target lesion revascularization; TVF: target vessel failure; TLF: target lesion failure.

*per protocol (please refer to study definition of $\mathrm{MI}$ in the methods section) 
Table 5. Cumulative two-year scaffold thrombosis

\begin{tabular}{|c|c|c|c|c|}
\hline Scaffold thrombosis (\%) & $\begin{array}{l}\text { Total population } \\
\mathrm{N}=812 \text { pts }\end{array}$ & $\begin{array}{l}\text { OVP scaffolds } \\
\mathrm{N}=115 \text { pts }\end{array}$ & $\begin{array}{c}\text { Non-OVP scaffolds } \\
\mathrm{N}=697 \text { pts }\end{array}$ & $P$-value \\
\hline \multicolumn{5}{|l|}{$0-30$ days } \\
\hline - Early definite & $4(0.5)$ & $1(0.9)$ & $3(0.4)$ & 0.5 \\
\hline - Early definite/probable & $5(0.6)$ & $2(1.7)$ & $3(0.4)$ & 0.1 \\
\hline \multicolumn{5}{|l|}{$31-365$ days } \\
\hline - Late definite & $3(0.4)$ & 0 & $3(0.4)$ & 1.0 \\
\hline - Late definite/probable & $3(0.4)$ & 0 & $6(0.9)$ & 1.0 \\
\hline \multicolumn{5}{|l|}{ 366-758 days } \\
\hline Very Late definite & $4(0.5)$ & 0 & $3(0.4)$ & 1.0 \\
\hline Very Late definite/probable & $4(0.5)$ & 0 & $4(0.6)$ & 1.0 \\
\hline
\end{tabular}

OVP: overlapping. 


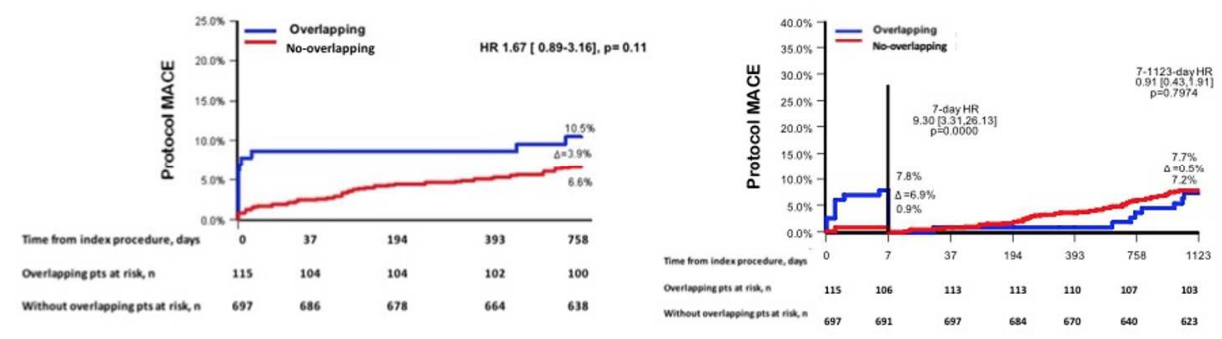

Figure 1. Cumulative incidence of MACE in patients treated with and without ABSORB overlapping. On the left the two-year curve for both groups and, on the right, the landmark analysis with the in hospital events.

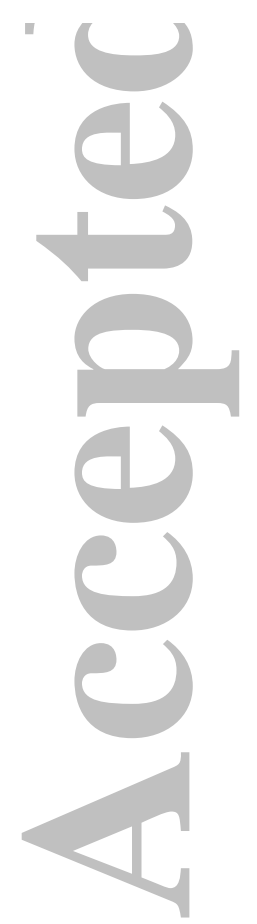

$338 \times 166 \mathrm{~mm}(300 \times 300$ DPI $)$ 


\section{(1)}

Two-year Clinical Outcomes of Patients Treated with Overlapping Absorb

\section{Scaffolds: An Analysis of the ABSORB EXTEND Single-Arm Study}

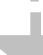

J Ribamar Costa Jr. MD ${ }^{1}$, Alexandre Abizaid MD, $\mathrm{PhD}^{1,2}$; Antonio L. Bartorelli $\mathrm{MD}^{3}$, Robert Whitbourn MD ${ }^{4}$ Patrick W. Serruys ${ }^{5}, \mathrm{MD}, \mathrm{PhD}$; Pieter C. Smits, $\mathrm{MD}^{6}$ on behalf of the ABSORB EXTEND investigators

${ }^{1}$ Instituto de Cardiologia Dante Pazzanese, Sao Paulo, Brazil ; ${ }^{2}$ Hospital Sirio Libanes, , Sao Paulo, Brazil,

${ }^{3}$ Centro Cardiologico Monzino, IRCCS, University of Milan, Milan, Italy, ${ }^{4} \mathrm{St}$ Vincent's Hospital, Fitzroy, Victoria, Australia, ${ }^{5}$ International Centre for Circulatory Health, NHLI, Imperial College London, London, UK ${ }^{6}$ Maasstad Hospital, Rotterdam, The Netherlands

Short title: Outcomes of ABSORB in overlapping

Conflict of interest: None of the authors have conflict to declare

Word count: 4463

\section{Correspondence to:}

Alexandre Abizaid., MD, PhD, FACC

Av. Dr. Dante Pazzanese, 500 - Vila Mariana - São Paulo, SP, Brasil

CEP 04012-180

e-mail: aabizaid@uol.com.br 


\section{ABSTRACT}

Background: Pre-clinical data showed that overlapping (OVP) scaffolds might result in delayed healing and strut coverage compared to non-OVP scaffold segments. Furthermore, OVP in patients could result in increased peri-procedure myocardial infarction (MI) rate secondary to side branch occlusion; however, little is known whether this may have an impact on long-term clinical outcomes.

Methods: ABSORB EXTEND is a prospective, single-arm, open-label clinical study in which 812 patients were enrolled at 56 sites. In the present study, we compared the immediate and two-year clinical outcomes of patients with OVP scaffolds $(n=115)$ to those of patients with non-OVP scaffolds $(n=697)$. The primary objective was the comparison of MACE (cardiac death, MI and ischemic-driven TLR) and scaffold thrombosis (ST) rates up to 2 years.

Results: Baseline clinical and angiographic characteristics were comparable between cohorts except for longer lesions in the OVP patients as expected $(16.7 \pm 7.3$ vs. $11.6 \pm 4.4 \mathrm{~mm}$, p $<0.0001$ ), higher lesion complexity (B2) and numerically smaller vessel size. In-hospital, there was a marked increase in MACE in the OVP cohort (7.0\% vs. $0.9 \%, p=0.002)$, exclusively driven by a higher rate of peri-procedure MI $(7.0 \%$ vs. $0.9 \%, p=0.002)$. Longterm MACE did not significantly differ between groups (10.4\% in the OVP cohort vs. $6.6 \%$ in the no-OVP group, $\mathrm{p}=0.1)$ with comparable rates of cardiac death $(0.9 \%$ vs. $1.2 \%, \mathrm{p}=1.0)$ and ischemia-driven TLR (1.7\% vs. $2.5 \%, \mathrm{p}=1.0)$. Cumulative incidence of MI was higher in the OVP cohort $(7.8 \%$ vs. $3.0 \%, p=0.02)$. Of note, the rate of MI between hospital discharge and two-year follow-up was lower in the OVP cohort $(0.8 \%$ vs. $2.1 \%, p=0.04)$. Cumulative incidence of definite/probable ST was relatively low and comparable between groups $(1.8 \%$ vs. $1.5 \%, \mathrm{p}=0.7)$.

Conclusions: In this low-to-moderate complex population treated with the ABSORB scaffold the OVP group showed a higher incidence of peri-procedure MI with no immediate or long-term increase in cardiac death, TLR or ST.

Key words: ABSORB, overlapping, MACE, single arm study. 


\section{Introduction}

It is well established in the literature that metallic stent overlapping (OVP) is associated with higher peri-procedural myocardial infarction (MI), probably due to more side branch occlusion along the treated segment, an event linked also to the device design and strut thickness and width ${ }^{1,2}$.

The advent of metallic drug-eluting stents (DES) increased the concern related to the double layer of polymer and double dose of the anti-proliferative drug accrued in the OVP segments. Finn et al. analyzed rabbit models treated with $1^{\text {st }}$-generation DES (Cypher ${ }^{\mathrm{TM}}$ [Cordis Corporation, NJ, USA] and Taxus ${ }^{\mathrm{TM}}$ [Boston Scientific, MA, USA]) and their analogous bare-metal stents $\left(B x V e l o c i t y{ }^{\mathrm{TM}}\right.$ [Cordis Corporation] and Express ${ }^{\mathrm{TM}}$ [Boston Scientific]) and showed that, compared with bare-metal stents, $1^{\text {st }}$-generation DES further delay arterial healing and promote inflammation at sites of $\mathrm{OVP}^{3}$. Newer-generation DES, with thinner struts, open-cell design and more biocompatible or even biodegradable polymer coatings, contributed to minimize the negative impact of OVP and have been proven to be safer than $1^{\text {st }}$-generation $\mathrm{DES}^{4,5}$.

In theory, the recent introduction of BVS has made OVP more attractive, since these devices will be ultimately absorbed and the functionality of the treated segment should be potentially restored. Nonetheless, it is important to keep in mind that the current generation of BVS is still bulky with strut thickness and width of about150 $\mu \mathrm{m}$ and $200 \mu \mathrm{m}$, respectively, which are higher than those of the $1^{\text {st }}$-generation DES and may affect acute and long-term outcomes of this novel technology. In the present manuscript, we sought to compare acute and two-year outcomes of patients treated with ABSORB BVS with and without OVP scaffolds.

\section{Methods}

\section{Study Design and Population}

In the present analysis, we included all patients enrolled in the ABSORB EXTEND study. For comparison purposes, the entire population was divided according to the presence or not of OVP.

The details of the ABSORB EXTEND trial have been described elsewhere ${ }^{6}$. In brief, this was a prospective, single-arm, open-label clinical trial that enrolled 812 patients at 56 
international sites outside of the US. Target arteries should have a reference vessel diameter (RVD) $\geq 2.0 \mathrm{~mm}$ and $\leq 3.8 \mathrm{~mm}$, a maximum lesion length of $\leq 28 \mathrm{~mm}$, a diameter stenosis $\geq 50 \%$ and $<100 \%$. A maximum of two de novo native coronary artery lesions could be treated, each located in a different major epicardial vessel. Major exclusion criteria included a recent MI ( $<72$ hours before the index procedure) and target lesions located in the left main or within an arterial or saphenous vein graft. Also excluded were lesions with excessive tortuosity and/or heavy calcification.

Abbott Vascular (Santa Clara, CA) funded the study. The research ethics committee of each participating institution approved the protocol and all enrolled patients provided written informed consent before inclusion. The study is registered on clinicaltrials.gov (unique identifier NCT01023789).

\section{Study Device}

The study device (Abbott Vascular, Santa Clara, CA) is the same as that used in the ABSORB Cohort B trial, and has been described in detail previously ${ }^{7,8}$. In brief, the balloon expandable Absorb BVS is comprised of a poly L-lactide (PLLA) backbone, coated with a matrix composed of the antiproliferative drug everolimus (Certican ${ }^{\circledR}$ Novartis Pharmaceuticals Corporation, Basel, Switzerland) and the polymer poly (D-lactide) (PDLLA) in a 1:1 ratio to form an amorphous drug-eluting coating matrix containing $100 \mu \mathrm{g}$ everolimus $/ \mathrm{cm}^{2}$. Both PLLA and PDLLA are fully bioresorbable; PDLLA is expected to be completely absorbed by the body in 9 months and PLLA in approximately 24-36 months. During the resorption process, ester bonds in the PLLA and PDLLA chains are hydrolyzed, and small particles ( $\leq 2 \mu \mathrm{m}$ in diameter) are phagocytosed by macrophages. The ultimate degradation product of both PLLA and PDLLA is lactic acid, which is biologically ubiquitous and metabolized via the Krebs cycle?.

\section{Study Procedure}

Patients were enrolled through an interactive voice response system (Oracle America, Inc. Woburn, MA), following confirmation of angiographic inclusion criteria and delivery of the Absorb BVS device beyond the guiding catheter. Enrolled patients were to remain in the study until completion of the required follow-up period.

A maximum of two de novo native coronary artery lesions could be treated, each located in a different major epicardial vessel. The recommended range for target vessel diameter was assessed in terms of online quantitative coronary angiography (QCA) or 
intravascular ultrasound parameters of distal Dmax and proximal Dmax, which refer to the maximum lumen diameter evaluated at the distal and proximal ends of the target segment to be scaffolded, respectively. Planned OVP of scaffolds was permitted in lesions $>22 \mathrm{~mm}$ and $\leq 28 \mathrm{~mm}$ in length, with an OVP ranging between $1 \mathrm{~mm}$ and $4 \mathrm{~mm}$. Although not mandatory, the "scaffold-to-scaffold" implantation technique was recommended to minimize OVP,

which was achieved by placing the balloon marker of the proximal scaffold just before the scaffold marker of the distal scaffold ${ }^{10}$.

All target lesions were to be treated using standard interventional techniques with mandatory predilation and scaffold implantation at a pressure not exceeding the balloon rated burst pressure. Postdilatation was left to the discretion of the investigator; however, if performed, was to be done with a non-compliant balloon sized to fit within the boundaries of the scaffold $(\leq 0.5 \mathrm{~mm}$ of the nominal scaffold diameter).

All patients enrolled in the study were to be pre-treated with a loading dose of $\geq 300$ $\mathrm{mg}$ of clopidogrel and $\geq 300 \mathrm{mg}$ of aspirin, followed by $75 \mathrm{mg}$ of clopidogrel daily for a minimum of 6 months and $\geq 75 \mathrm{mg}$ of aspirin daily indefinitely.

\section{Source document verification}

Source document verification (SDV) was routinely performed in $100 \%$ of all reported events and $100 \%$ of patients through 30-day follow-up. Subsequently, SDV was performed in a random $20 \%$ of patients for the remaining follow-up visits.

\section{Follow-up}

Assessment of anginal status, data collection of adverse events, details of any subsequent coronary interventions, and use and changes in concomitant medications were collected at 30 days ( \pm 7 days), 180 days ( \pm 14 days) and at 1 and 2 years ( \pm 28 days).

\section{Study Endpoints and Definitions}

In addition to acute success, which is comprised of clinical device (analyzed on a per lesion basis) and clinical procedure success (analyzed on a per subject basis), endpoints include the comparison of adjudicated scaffold thrombosis (ST), cardiac death, MI (target and non-target vessel), and revascularization (target lesion revascularization [TLR]/target 
vessel revascularization [TVR]/all revascularizations) rates. The composite rates of ischemiadriven major adverse cardiac events (ID-MACE), ischemia-driven target vessel failure (IDTVF), ischemia-driven target lesion revascularization (ID-TLR) and ischemia-driven target vessel revascularization (ID-TVR) were also compared between patients with and without OVP.

An independent Clinical Event Committee (CEC) adjudicated all study endpoint events according to either protocol definitions and/or the Academic Research Consortium (ARC) definitions. All adverse events were reported to an independent Data and Safety Monitoring Board (DSMB), which reviewed the data to identify any safety issues related to the conduct of the study.

Clinical device success was defined as successful delivery and deployment of the clinical investigation scaffold at the target lesion and successful withdrawal of the scaffold delivery system with attainment of a final residual stenosis $<50 \%$ by QCA or by visual estimation if QCA was unavailable. Standard predilation and postdilatation balloon catheters (if applicable) could be used. Bailout patients were included as device success only if the above criteria for clinical device success were met.

Clinical procedure success was defined as successful delivery and deployment of the clinical investigation scaffold at the target lesion and successful withdrawal of the scaffold delivery system with attainment of a final residual stenosis of $<50 \%$ by QCA or by visual estimation if QCA was unavailable, and/or using any adjunctive device without the occurrence of ID-MACE during hospital stay with a maximum of first seven days post index procedure. In a dual lesion setting, both lesions must have met clinical procedure success.

Cardiac death was defined as any death due to proximate cardiac cause (e.g., MI, lowoutput heart failure, fatal arrhythmia). Unwitnessed death and death of unknown cause were classified as cardiac death. This included all procedure-related deaths comprising those related to concomitant treatment.

Classification and criteria for MI diagnosis were defined according to the per protocol definition. Q-wave MI was the development of a new, pathological Q wave. Non-Q-wave MI was elevation of CK levels to $\geq$ two times the upper limit of normal with elevated CK-MB in the absence of new pathological Q waves.

Revascularization events were defined as: a) ID-MACE, composed of cardiac death, MI (Q-wave and non-Q-wave) and ID-TLR by CABG or PCI; b) ID-TVF, composed of cardiac death, MI (Q-wave and non-Q-wave); c) ID-TVR by CABG or PCI. 
ID-TLR was defined as any repeat percutaneous intervention of the target lesion or bypass surgery of the target vessel with either positive functional ischemia study, ischemic symptoms and angiographic minimal lumen diameter stenosis $\geq 50 \%$ by core laboratory QCA or revascularization of a target lesion with diameter stenosis $\geq 70 \%$ by core laboratory QCA without either ischemic symptoms or a positive functional study.

ID-TVR was defined as any repeat percutaneous intervention or surgical bypass of any segment of the target vessel with either positive functional ischemia study or ischemic symptoms and an angiographic minimal lumen diameter stenosis $\geq 50 \%$ by core laboratory or revascularization of a target vessel with diameter stenosis $\geq 70 \%$ by core laboratory QCA without either ischemic symptoms or a positive functional study.

ST was categorized as acute ( $<1$ day), subacute (1-30 days) and late ( $>30$ days), and was defined according to the ARC guidelines ${ }^{11}$ as follows: definite, acute coronary syndrome and angiographic or pathologic confirmation of scaffold thrombosis; probable, unexplained death $\leq 30$ days or target vessel MI without angiographic information; and possible, unexplained death $>30$ days after scaffold placement.

\section{Statistical Analysis}

The sample size for ABSORB EXTEND rather than being defined based on an endpoint hypothesis was chosen to provide information on device performance and should be seen as hypothesis generating. All analyses were performed on the per treatment evaluable population.

For the descriptive statistics, categorical data are presented as counts and percentages and continuous variables are presented as mean \pm standard deviation (SD). Categorical variables were compared by the Chi-square test or Fisher's test when the Cochran's rule was not met. Continuous variables were compared by the Student $t$ test. Time-to-event MACE is presented as a Kaplan-Meier curve.

To determine the independent predictors of MACE, TLR, MI and ST among patients treated with the ABSORB BVS, a multivariable logistic regression model was built using a stepwise (forward/backward) procedure, with independent variables entered into the model at the 0.20 significance level and removed at the 0.10 level. Variables were eligible for inclusion in the multivariable logistic regression model-building process if the variable was present for $90 \%$ of the subjects in the analyses, they had a p-value $<0.2$ from the univariable 
analysis, and, if they highly correlated with another variable $(r>0.5$ and $p<0.05)$, had the higher level of significance. Presence of OVP was included in the model.

A two-tailed $p$ value $<0.05$ was considered statistically significant. However, since $p$ values presented here are for exploratory analysis only, they should therefore be interpreted cautiously.

\section{RESULTS}

Of the 812 patients (874 lesions) enrolled in the ABSORB EXTEND study, 115 (128 lesions, 14.2\%) were treated with OVP. Patients had a mean age of 61 years and were predominantly men in both cohorts. Baseline clinical demographics (Table 1) did not significantly differ between patients with and without OVP, except for the initial clinical presentation, with stable coronary disease more prevalent among patients treated with OVP, while patients without OVP had more frequently acute coronary syndrome as the initial presentation.

Table 2 displays the relevant angiographic clinical characteristics. As expected, lesions in the OVP cohort were longer with a higher prevalence of increased complexity (B2 according to ACC/AHA classification) $(66 \%$ vs. $38 \%, \mathrm{p}<0.01)$. Predilation was performed in almost all patients in both cohorts, while postdilation was more frequently performed among patients with OVP (77.4\% vs. $68.2 \%, \mathrm{p}=0.05)$.

Procedure success was comparable between the cohorts. However, clinical success was slightly higher among patients without OVP, mainly due to more peri-procedure MI detected among them $(94.3 \%$ vs. $90.4 \%, \mathrm{p}<0.001)$ (Table 3$)$.

Notably, if we consider the current definition of good scaffold implantation (PSP $=$ patients with pre-dilatation, and QCA RVD $\geq 2.25 \mathrm{~mm}-\leq 3.5 \mathrm{~mm}$, and post-dilatation performed at $\geq 18$ atm, with post-dilatation balloon diameter $>$ nominal scaffold diameter but $\leqq$ nominal scaffold diameter $+0.5 \mathrm{~mm}$ ), this was achieved in only $20 \%$ of the OVP cohort and $13.7 \%$ of the control group ( $\mathrm{p}=0.02)$

Incidence of other MACE components, including cardiac death and vessel revascularization as well as ST, TLF and TVF were comparable between groups at two-year follow-up (Tables 4 and 5). Of note, there was no case of definitive ST in the OVP cohort in the first year after the procedure, while in the non-OVP group a $1.1 \%$ rate was observed in the same period. 
Figure 1 shows the temporal distribution of MACE between the two groups. As noticed, most of the adverse events in the OVP population occurred within the in-hospital period.

There was no independent predictor of MACE in the overall population. However, a trend toward more events was observed among patients with small reference vessel diameter (OR 0.90 IC95\% [0.01, 1.04], $\mathrm{p}=0.054$ ) and in those with prior MI history (OR 3.18 IC 95\% $[0.93,10.84], p=0.064)$. The only independent predictor of MI was a prior history of MI (OR 5.15 , IC95\% [1.42, 18.70], $\mathrm{p}=0.012)$. Independent predictor of a new revascularization procedure was treatment of multivessel disease (OR 6.16, IC 95\% [1.72, 22.11], p=0.005). There were no independent predictors of ST or death.

\section{DISCUSSION}

To the best of our knowledge, this is the largest long-term follow-up of patients undergoing treatment with OVP scaffolds. The results of this study should be interpreted with caution due to the differences in baseline lesion characteristics between the two groups. The main findings are the following: 1) there was a higher incidence of peri-procedure MI in the OVP patients who, however, had longer lesions, numerically smaller RVD, and greater B2 lesion complexity and; 2) two-year MACE including cardiac death, ID-TLR and ST were similar between these low-to-moderate complexity patient groups.

Treatment of long lesions with BVS frequently require scaffold OVP, since the maximum length of the commercially available devices is $28 \mathrm{~mm}$. Development of longer devices, although possible, would face one of the current limitations of this technology. Since the device should not be postdilated more than $0.5 \mathrm{~mm}$ than its nominal diameter to prevent the risk of acute disruption, the development of longer scaffolds would make difficult to accommodate vessel tapering, which often results in differences $>0.5 \mathrm{~mm}$ between the proximal and distal reference vessel segment in the scenario of long lesions.

Furthermore, it is not always technically easy or feasible to advance such bulky devices into each other to achieve OVP segments, especially when treating tortuous, and/or calcified vessels or when an unplanned OVP at the distal edge of a previously implanted BVS is needed. Use of tension/pressure to advance the device may result in dislodgement and damage to its integrity.

Once OVP is achieved, a segment with a 300-400- $\mu$ m layer of polymer will be created, which might impair local flow dynamic ${ }^{12}$ and increase the risk of side branch occlusion. Muramatsu et al. have already demonstrated that deployment of the Absorb BVS 
without OVP was associated with a higher incidence of post procedural side-branch occlusion when compared with a thin-strut new-generation DES (Xience V, Abbott Vascular $)^{13}$. Despite the lack of abundant clinical data, we speculate that these findings may be aggravated in segments of double layers of BVS. In a recent publication of the ABSORB II trial, Ishibashi et al. reported that BVS OVP was the single independent predictor of per protocol peri-procedure MI (OR: 5.07, 95\% CI: 1.78 to $14.41, \mathrm{p}=0.002)^{14}$. A previous analysis of the ABSORB EXTEND study database pointed also to OVP as a predictor of peri-procedure $\mathrm{MI}^{15}$. The present analysis confirms this finding that is probably related to the occlusion of more side branches along the longer segments treated with OVP.

Regarding long-term outcomes, the presence of scaffold OVP has also been associated with negative findings, at least in the pre-clinical scenario. Farooq et al. examined 41 OVP performed with the ABSORB BVS or the everolimus-eluting metallic DES in pigs and showed that strut coverage at 28 days was delayed in the OVP scaffolds because of the overlay configuration of the thicker ABSORB BVS struts. However, at 90 days OVP of both ABSORB BVS and metallic DES showed comparable strut coverage ${ }^{16}$. It is noteworthy that the pre-clinical observation of delayed strut coverage did not translate into an increased occurrence of serious untoward events in our clinical evaluation. This might be related to adherence to the dual antiplatelet therapy (roughly $96 \%$ at 6 months and $79 \%$ at one year, for both groups), the frequent optimization of the procedure with postdilation (especially in the OVP group) and the low-to-moderate complexity of the enrolled population. Furthermore, in our present study, use of OVP also did not turn out as an independent predictor of MACE, TLF, MI, TLR or ST.

It is also important to bear in mind that this study was conceived and primarily conducted prior to the current recommendations for optimal scaffold deployment, including the endorsement for performing routine postdilation with high-pressure non-compliant balloons within the limits of expansion of the device, as recently published by Ortega-Paz et al. ${ }^{17}$.

Recently, Sotomi et al. published the results of 14 patients treated with ABSORB OVP and evaluated with OCT at two years ${ }^{18}$. They compared the results in the segments with and without OVP. Notably, lumen area and endoluminal scaffold areas were similar in both segments despite the neointimal area being larger in the overlap segments. The neointimal coverage was essentially fully complete in both non-overlap (99.4 $\pm 0.8 \%$ ) and overlap segments $(99.8 \pm 0.4 \%)$ at two-year follow-up. The flow area in the overlap segments was not different from the flow area in the non-overlap segments, despite the neointimal response 
being greater in the overlap segments. Consequently, the treated segments showed a homogeneous lumen area through the scaffold segment. These results might support the feasibility of overlapping scaffolds when needed for longer lesions if acute lumen expansion is achieved in a similar extent as in the non-overlap segments using good implantation techniques.

\section{LIMITATIONS}

This post-hoc analysis was performed from a non-randomized study. No correction has been made regarding differences in baseline characteristics between the two comparison groups. These differences could have heavily contributed to the results. The study was underpowered for low-frequency events, like ST. The implantation technique used in the ABSORB Extend was outdated. Indeed, the PSP (vessel predilation, sizing and postdilation)

implant strategy is highly recommended and followed today. Finally, the lack of a control arm with a metallic DES does not allow for comparison of the devices.

\section{CONCLUSIONS}

In this low-to-moderate complex population treated with the BVS in the ABSORB EXTEND study, scaffold OVP was associated with an increase of peri-procedure MI but without any immediate or long-term effect on cardiac death, TLR or ST occurrence.

\section{REFERENCES}

Ellis SG, Savage M, Fischman D et al. Restenosis after placement of PalmazSchatz stents in native coronary arteries. Initial results of a multicenter experience. Circulation 86(6), 1836-1844 (1992).

2. Kastrati A, Schömig A, Elezi S et al. Predictive factors ofrestenosis after coronary stent placement. J. Am. Coll. Cardiol.30(6), 1428-1436 (1997).

3. Finn AV, Kolodgie FD, Harnek $J$ et al. Differential response of delayed healing and persistent inflammation at sites of overlapping sirolimus- or paclitaxel-eluting stents. Circulation 112(2), 270-278 (2005).

4. Farooq V, Vranckx P, Mauri L et al. Impact of overlapping newer generation drug-eluting stents on clinical and angiographic outcomes: pooled analysis of five trials from the international Global RESOLUTE Program. Heart 99(9), 626-633 (2013). 
5. Kitabata H, Loh JP, Pendyala LK et al. Safety and efficacy outcomes of overlapping second-generation everolimus-eluting stents versus first-generation drug-eluting stents. Am. J.Cardiol. 112(8), 1093-1098 (2013).

Abizaid A, Ribamar Costa J Jr, Bartorelli AL, Whitbourn R, van Geuns RJ, Chevalier B, Patel T, Seth A, Stuteville M, Dorange C, Cheong WF, Sudhir K, Serruys PW; ABSORB EXTEND investigators. The ABSORB EXTEND study: preliminary report of the twelve-month clinical outcomes in the first 512 patients enrolled. EuroIntervention. 2015 Apr;10(12):1396-401.

7. Oberhauser JP, Hossainy S, Rapoza R. Design principles and performance of bioresorbable polymeric vascular scaffolds. EuroIntervention. 2009;Vol.5:F15-F22

8. Okamura T, Garg S, Gutierrez-Chico JL, Shin ES, Onuma Y, Garcia-Garcia HM, Rapoza RJ, Sudhir K, Regar E, Serruys PW. In vivo evaluation of stent strut distribution patterns in the bioabsorbable everolimus-eluting device: An oct ad hoc analysis of the revision 1.0 and revision 1.1 stent design in the absorb clinical trial. EuroIntervention. 2010;5:932-938

9. Serruys PW, Onuma Y, Ormiston JA, de Bruyne B, Regar E, Dudek D, Thuesen L, Smits PC, Chevalier B, McClean D, Koolen J, Windecker S, Whitbourn R, Meredith I, Dorange C, Veldhof S, Miquel-Hebert K, Rapoza R, García-García HM. Evaluation of the second generation of a bioresorbable everolimus drug-eluting vascular scaffold for treatment of de novo coronary artery stenosis: six-month clinical and imaging outcomes. Circulation. 2010 Nov 30;122(22):2301-12.

10. Rigatelli G, Avvocata FD, Ronco F, Giordan M, Roncon L, Caprioglio F, Grassi G, Faggian G, Cardaioli P. Edge-to-Edge Technique to Minimize Ovelapping of Multiple Bioresorbable Scaffolds Plus Drug Eluting Stents in Revascularization of Long Diffuse Left Anterior Descending Coronary Artery Disease. J Interv Cardiol. 2016 Jun;29(3):275-84.

11. Cutlip D, Windecker S, Mehran R, Boam A, Cohen D, van Es G-A, Steg P, Morel M-A, Mauri L, Vranckx P, McFadden E, Lansky A, Hamon M, Krucoff M, Serruys P, Consortium aobotAR. Clinical end points in coronary stent trials. A case for standardized definitions. Circulation. 2007;115:2344-2351

12. Rikhtegar F, Wyss C, Stok KS, Poulikakos D, Müller R, Kurtcuoglu V. Hemodynamics in coronary arteries with overlapping stents. J. Biomech. 47(2), 505-511 (2014). 
13. Muramatsu T, Onuma Y, García-García HM et al.; ABSORB EXTEND Investigators. Incidence and short-term clinical outcomes of small side branch occlusion after implantation of an everolimus-eluting bioresorbable vascular scaffold: an interim report of 435 patients in the ABSORB-EXTEND single-arm trial in comparison with an everolimuseluting metallic stent in the SPIRIT first and II trials. JACC Cardiovasc. Interv. 6(3), 247257 (2013).

14. Ishibashi Y, Muramatsu T, Nakatani S, Sotomi Y, Suwannasom P, Grundeken MJ, Cho YK, Garcia-Garcia HM, van Boven AJ, Piek JJ, Sabaté M, Helqvist S, Baumbach A, McClean D, de Sousa Almeida M, Wasungu L, Miquel-Hebert K, Dudek D, Chevalier B, Onuma Y, Serruys PW. Incidence and Potential Mechanism(s) of Post-Procedural Rise of Cardiac Biomarker in Patients With Coronary Artery Narrowing After Implantation of an Everolimus-Eluting Bioresorbable Vascular Scaffold or Everolimus-Eluting Metallic Stent. JACC Cardiovasc Interv. 2015 Jul;8(8):1053-63.

15. De Ribamar Costa Jr J, Abizaid A, Bartorelli A, Whitbourn R, Jepson N, Perin M, Steinwender C, Stuteville M, Ediebah D, Sudhir K, Serruys PW. One-year clinical outcomes of patients treated with everolimus-eluting bioresorbable vascular scaffolds versus everolimus-eluting metallic stents: a propensity score comparison of patients enrolled in the ABSORB EXTEND and SPIRIT trials. EuroIntervention 2016;12:1255-1262

15. Farooq V, Serruys PW, Heo JH et al. Intracoronary optical coherence tomography and histology of overlapping everolimus-eluting bioresorbable vascular scaffolds in a porcine coronary artery model: the potential implications for clinical practice. JACC Cardiovasc. Interv. 6(5), 523-532 (2013).

17. Ortega-Paz L, Capodanno D, Gori T, Nef H, Latib A, Caramanno G, Di Mario C, Naber C, Lesiak M, Capranzano P, Wiebe J, Mehilli J, Araszkiewicz A, Pyxaras S, Mattesini A, Geraci S, Naganuma T, Colombo A, Münzel T, Sabaté M, Tamburino C, Brugaletta S. Predilation, sizing and post-dilation scoring in patients undergoing everolimuseluting bioresorbable scaffold implantation for prediction of cardiac adverse events: development and internal validation of the PSP score. EuroIntervention. 2017 Apr 20;12(17):2110-2117.

18. Sotomi Y, Suwannasom P, Wu CJ, Tateishi H, Cheong WF, Zhao WF, Veldhof S, Winter RJ, Wykrzykowska J, Farooq V, Abizaid A, Serruys PW, Onuma Y. Imaging outcomes of bioresorbable scaffold overlap: an optical coherence tomography analysis from the ABSORB EXTEND trial. AsiaIntervention 2017;3:49-57. 


\section{Figure Legend}

Figure 1. Cumulative incidence of MACE in patients treated with and without ABSORB overlapping. On the left the two-year curve for both groups and, on the right, the landmark analysis with the in hospital events.
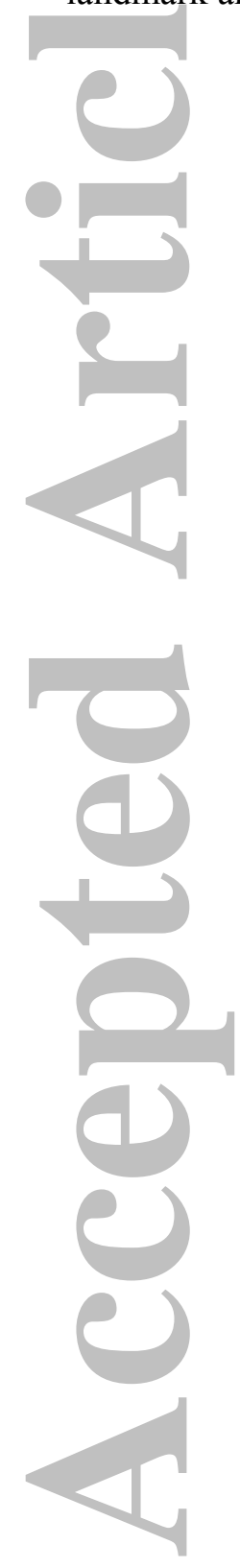


\section{University Library}

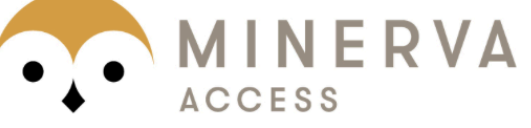

A gateway to Melbourne's research publications

Minerva Access is the Institutional Repository of The University of Melbourne

Author/s:

Ribamar Costa, J;Abizaid, A;Bartorelli, AL;Whitbourn, R;Serruys, PW;Smits, PC

Title:

Two-year clinical outcomes of patients treated with overlapping absorb scaffolds: An analysis of the ABSORB EXTEND single-arm study

Date:

2018-06-01

Citation:

Ribamar Costa, J., Abizaid, A., Bartorelli, A. L., Whitbourn, R., Serruys, P. W. \& Smits, P. C. (2018). Two-year clinical outcomes of patients treated with overlapping absorb scaffolds: An analysis of the ABSORB EXTEND single-arm study. CATHETERIZATION AND CARDIOVASCULAR INTERVENTIONS, 91 (7), pp.1202-1209. https://doi.org/10.1002/ ccd.27223.

Persistent Link:

http://hdl.handle.net/11343/293563 